\title{
Availability of Software-Based Correction of Mandibular Plane for the Vertical Measurement of the Mandible in Cone Beam Computed Tomography
}

\author{
Sang-Sun Han, ${ }^{1}$ Kwang-Min Lee, ${ }^{2}$ and Kee-Deog Kim $^{2}$ \\ ${ }^{1}$ Department of Oral and Maxillofacial Radiology, College of Dentistry, Yonsei University, Seoul 03722, Republic of Korea \\ ${ }^{2}$ Department of Advanced General Dentistry, College of Dentistry, Yonsei University, Seoul 03722, Republic of Korea \\ Correspondence should be addressed to Kee-Deog Kim; kdkim@yuhs.ac
}

Received 10 September 2015; Accepted 8 October 2015

Academic Editor: Guang Jia

Copyright (C) 2015 Sang-Sun Han et al. This is an open access article distributed under the Creative Commons Attribution License, which permits unrestricted use, distribution, and reproduction in any medium, provided the original work is properly cited.

Objectives. To investigate the availability of correction of mandibular plane using software for vertical measurements in cone beam computed tomography (CBCT) according to the sites of the mandible. Methods. CBCT scans of six dry mandibles were performed at 0-, 5-, 10-, 15-, and 20-degree angles relative to CBCT scanning table. Using the imaging software, mandibular planes of the different angles were corrected to that of 0-degree angle on the CBCT images. Before and after correction of the mandibular planes, the distance from the mandibular canal to the alveolar crest was measured at M1, M2, and M3 areas of the mandible and vertical measurements were statistically compared with those of 0 -angle location using the paired $t$-test. Results. Prior to correction, the vertical measurements increased as the angle increased. The greatest differences of measurements were observed in M3 areas $(P<$ 0.05). After correction, a strong correlation was found in measurements between the 0-degree angle and the other angles in all sites of the mandible $(P>0.05)$. Conclusions. The vertical measurements of CBCT were significantly influenced by mandibular positioning. When CBCT scans are performed at angles other than 0-degree angle, software-based correction of the mandibular plane can be a reliable tool for the accurate vertical measurements in CBCT.

\section{Introduction}

The assessment of the available bone height is one of the significant factors which influences the decision regarding the length of the implant prior to dental implant placement [1]. Computed tomography (CT) is an accurate imaging modality for the evaluation of preimplant sites in the mandibles [2]. Cone beam computed tomography (CBCT) also enables measurement of the distance between the alveolar crest and the mandibular canal so that impingement of the inferior alveolar nerve can be avoided [3-6].

Recently, due to the advantages of low radiation exposure and relatively low cost, $\mathrm{CBCT}$ scans have come to be preferred over CT for evaluations of bone quantity prior to dental implant placement $[4,7,8]$. Additionally, CBCT is known to provide measurements with submillimeter accuracy [9].

Our previous study reported that vertical measurements based on CT scans can be significantly influenced by mandibular positioning angle [10]. In CBCT, the accuracy of the measurements is affected by the CBCT system and software, patient motion during the scan, and the clinician's skill in interpreting the images [3]. However, we were unable to identify any study that focused on the influence of changes in the mandibular position on the vertical measurements from CBCT scans.

An imaging software program has been developed to improve the applicability of $\mathrm{CBCT}$ imaging for dental treatments $[8,11,12]$. Today, this software program has the functionality to make the adjustment of the axes of CBCT data that are obtained at angles other than the 0 -degree angle. We thought that such functionality would make it possible to obtain the accurate vertical measurements regardless of the mandibular positions. However, no study has addressed the correction of mandibular plane using the software.

This study aimed at evaluating the influence of mandibular position changes on the vertical measurements from 


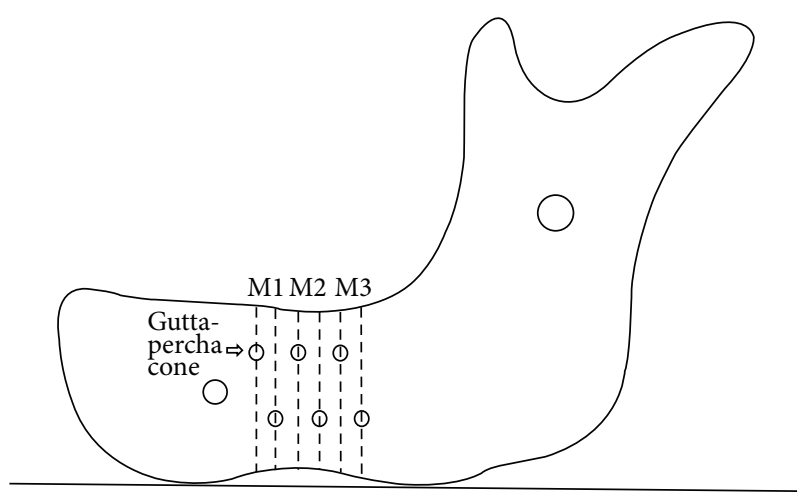

FIGURE 1: Marked sites of the mandible for the measurements.

CBCT scans and investigating the availability of softwarebased correction of mandibular plane on CBCT images for vertical measurements according to the sites of the mandible.

\section{Materials and Methods}

2.1. CBCT Scans. The CBCT scans were conducted with reference to the experimental procedure that we have previously reported for CT scans [10]. Six dry mandibles in partially edentulous states were used. To evaluate the measurement differences according to sites of the mandibles, gutta-percha cones $(1 \times 1 \mathrm{~mm})$ were attached as references to the areas at points M1 (5 and $10 \mathrm{~mm}$ distal to the mental foramen), M2 (15 and $20 \mathrm{~mm}$ distal to the mental foramen), and M3 (25 and $30 \mathrm{~mm}$ distal to the mental foramen) on the right and left buccal surfaces of the mandibles (Figure 1). To ensure the reproducibility of the $\mathrm{CBCT}$ scans with regard to different angles, the inferior border of the mandible was set on a $30 \mathrm{~mm}$ thick styrofoam plate that was fixed to an acrylic plate. To evaluate the influence of mandibular positional changes on the vertical measurements, the CBCT scans were performed in the positions described below.

The inferior border of the mandible (mandibular plane) was positioned parallel to the CBCT scanning table of 0 degree location and at the following positions:

(1) At a positive 5-degree angle to the scanning table (5degree location).

(2) At a positive 10-degree angle to the scanning table (10degree location).

(3) At a positive 15-degree angle to the scanning table (15degree location).

(4) At a positive 20-degree angle to the scanning table (20-degree location).

An Alphard 3030 CBCT unit (ASAHI Co., Tokyo, Japan) was used. All images were recorded at $80 \mathrm{kVp}$ and $5 \mathrm{~mA}$ over $17 \mathrm{~s}$ using a $102 \times 102 \mathrm{~mm}$ field of view and an axial slice thickness of $0.2 \mathrm{~mm}$. To obtain accurate results, the images of the remnant teeth and the extraction sockets were excluded.

2.2. Vertical Measurements of the CBCT Images before Correction of the Mandibular Plane. Before the correction of the

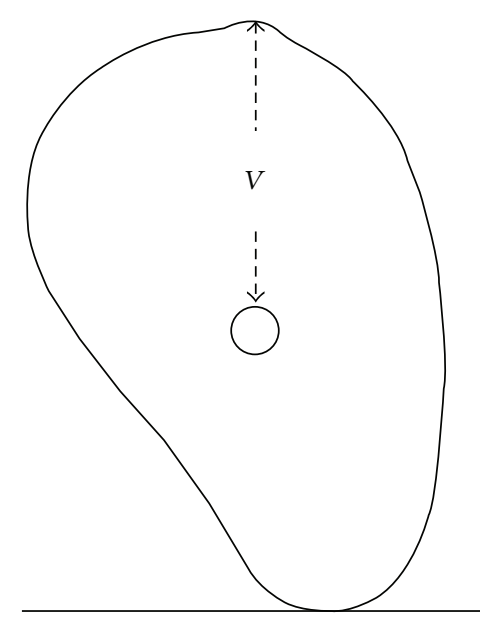

Figure 2: Measurement of the distance $(V)$ from the top of the mandibular canal to the alveolar crest on cross-sectional image.

mandibular plane, a total of 56 areas (18 M1 areas, $20 \mathrm{M} 2$ areas, and $18 \mathrm{M} 3$ areas) were obtained, and the 280 cross-sectional images taken from the 56 areas at 0-, 5-, 10-, 15-, and 20degree angles were used.

Using the In2Guide software OnDemand3D (Cybermed Inc., Seoul, Korea), the distances from the top of the mandibular canal to the alveolar crest were measured on the cross-sectional images at the marked areas at which the guttapercha was highly visible (M1, M2, and M3) at the 0-, 5-, 10-, 15-, and 20-degree angles (Figure 2). All measurements from $\mathrm{CBCT}$ images were performed twice with an interval of three weeks by a single experienced oral and maxillofacial radiologist and the means of these measurements were adopted for analysis.

2.3. Vertical Measurements of the CBCT Images after Correction of the Mandibular Plane. The imaging software OnDemand3D has the functionality to make the adjustment of the axes of CBCT data. This function is used for correction of the mandibular planes of CBCT images that are obtained at angles other than the 0-degree angle. In the adjustment of CBCT data, the base plane is dragged to reslice as newly aligned DICOM data. Rotation degrees will be shown on the $3 \mathrm{D}$ plane automatically. The mandibular planes in the $\mathrm{CBCT}$ images taken at the 5-, 10-, 15-, and 20-degree angles were corrected to that of the 0 -degree position for M1, M2, and M3 areas of the mandibles using the software program (Figure 3) and 224 cross-sectional images were added. The vertical measurements were performed using the same method that was applied to the CBCT images prior to correction.

2.4. Statistical Analyses. To assess intraobserver difference, Wilcoxon matched-pairs test was used for repeated measurements of the same observer. All vertical measurements before and after correction were statistically compared with those obtained at 0 -degree location according to the M1, M2, and M3 areas using the paired $t$-test $(P<0.05)$. The data set was analyzed using the Statistical Package for Social Science software ver. 19.0 (SPSS, Chicago, IL). 
TABle 1: Means and standard deviations of vertical measurements according to mandibular angles before correction ( $\mathrm{mm}$ ).

\begin{tabular}{lccccc}
\hline & $0^{\circ}$ & $5^{\circ}$ & $10^{\circ}$ & $15^{\circ}$ & $20^{\circ}$ \\
\hline M1 $(n=18)$ & $11.79 \pm 1.44$ & $11.84 \pm 1.43$ & $11.98 \pm 1.38$ & $12.12 \pm 1.36$ & $12.27 \pm 1.46$ \\
M2 $(n=20)$ & $10.12 \pm 1.30$ & $10.23 \pm 1.30$ & $10.56 \pm 1.49$ & $11.04 \pm 1.54$ & $11.34 \pm 1.75$ \\
M3 $(n=18)$ & $9.22 \pm 1.64$ & $9.45 \pm 1.64$ & $9.93 \pm 1.87$ & $10.52 \pm 1.95$ & $11.00 \pm 2.12$ \\
\hline
\end{tabular}

TABLE 2: Means and standard deviations of vertical measurements according to mandibular angles after correction (mm).

\begin{tabular}{lccccc}
\hline & $0^{\circ}$ & $5^{\circ}$ & $10^{\circ}$ & $15^{\circ}$ & $20^{\circ}$ \\
\hline M1 $(n=18)$ & $11.79 \pm 1.44$ & $11.77 \pm 1.51$ & $11.78 \pm 1.53$ & $11.83 \pm 1.46$ & $11.76 \pm 1.41$ \\
M2 $(n=20)$ & $10.12 \pm 1.30$ & $10.21 \pm 1.28$ & $10.17 \pm 1.32$ & $10.18 \pm 1.28$ & $10.21 \pm 1.28$ \\
M3 $(n=18)$ & $9.22 \pm 1.64$ & $9.18 \pm 1.74$ & $9.19 \pm 1.78$ & $9.25 \pm 1.71$ & $9.25 \pm 1.67$ \\
\hline
\end{tabular}

TABLE 3: Mean error and standard deviation between measurements at $0^{\circ}$ location and others before correction $(\mathrm{mm})$.

\begin{tabular}{ccccc}
\hline & $5^{\circ}$ & $10^{\circ}$ & $15^{\circ}$ & $20^{\circ}$ \\
\hline M1 $(n=18)$ & $0.04 \pm 0.24$ & $0.18 \pm 0.48$ & $0.29 \pm 0.96$ & $0.33 \pm 0.58$ \\
M2 $(n=20)$ & $0.10 \pm 0.26$ & $0.21 \pm 0.55$ & $0.91 \pm 0.45^{*}$ & $1.21 \pm 0.69^{*}$ \\
M3 $(n=18)$ & $0.19 \pm 1.05$ & $0.71 \pm 0.54^{*}$ & $1.31 \pm 0.71^{*}$ & $1.79 \pm 0.94^{*}$ \\
\hline
\end{tabular}

${ }^{*}$ Statistically significant difference at $P<0.05$.

TABLE 4: Mean error and standard deviation between measurements at $0^{\circ}$ location and others after correction $(\mathrm{mm})$.

\begin{tabular}{ccccc}
\hline & $5^{\circ}$ & $10^{\circ}$ & $15^{\circ}$ & $20^{\circ}$ \\
\hline $\mathrm{M} 1(n=18)$ & $0.02 \pm 0.21$ & $0.02 \pm 0.29$ & $0.04 \pm 0.20$ & $0.04 \pm 0.25$ \\
$\mathrm{M} 2(n=20)$ & $0.09 \pm 0.20$ & $0.04 \pm 0.18$ & $0.06 \pm 0.20$ & $0.09 \pm 0.24$ \\
$\mathrm{M} 3(n=18)$ & $0.04 \pm 0.32$ & $0.03 \pm 0.32$ & $0.03 \pm 0.27$ & $0.03 \pm 0.23$ \\
\hline
\end{tabular}

\section{Results}

There was no statistically significant intraobserver difference in repeated measurements of CBCT images $(P>0.05)$. Intraobserver consistency was rated at $95 \%$ between two measurements.

Before the correction of the mandibular plane, the value of vertical measurements increased as the angle between the mandibular plane and the scanning table increased (Table 1). The vertical measurements between the 0-degree and 5degree angles were not statistically significant different in any site of the mandible $(P>0.05)$. However, at the 15-degree and 20-degree angles, there were statistically significant differences for the M2 and M3 areas $(P<0.05$; Table 3$)$.

The differences of vertical measurements were more pronounced in the M3 areas than in the M2 areas and the differences in M2 areas were greater than those in M1 areas (Table 3). In the M3 areas, statistically significant differences at 10-, 15-, and 20-degree angles were observed $(P<0.05)$. However, in the M1 area, there were no statistically significant differences between 0 -degree angle and the other angles $(P>$ 0.05 ; Table 3).

In contrast, after the correction of the mandibular plane with software, the vertical measurements were relatively constant across different angles of the mandibular planes regardless of sites of the mandible (Table 2). There was no statistically significant difference in the measurements between the 0 -degree angle and the other angles $(P>0.05)$. Regarding the marked areas of the mandible, there was no statistically significant effect in the M1, M2, and M3 areas $(P>0.05$; Table 4$)$.

\section{Discussion}

During evaluations of preimplant sites of the mandible, accurate measurements of the distance from the mandibular canal to the alveolar crest on radiographs have been linked to primary implant success [13]. Insertion of an inadequately long implant can injure the inferior alveolar nerve resulting in permanent hypoesthesia of the lower lip [14].

Prior to the correction of mandibular planes, the vertical measurements based on CBCT scans were affected by changes in the position of the mandible. As the angle between the mandibular plane and the CBCT scanning table increased, the vertical measurements increased. Because the cross sections of CBCT images that were taken at angles other than the 0-degree angle were not perpendicular to the long axis of the mandible, the vertical measurements from the CBCT images taken at different angles might be overestimated compared to those at 0 -degree angle location of the mandible.

Regarding the sites of the mandible, before correction, the greatest differences of vertical measurements were observed in the posterior regions, and the difference of measurements decreased toward the more anterior regions. Because the M3 areas are the farthest from the axis of rotation in terms of angle, the cross-sectional images of the areas were oblique to the long axis of the mandible. So the measurements in the M3 areas were likely greater than those of the other sites in the mandibles.

Large errors in measurements of available bone height might cause nerve injury during the insertion of the implant. When patients are not accurately positioned in CBCT scans, CBCT rescans might be necessary for accurate evaluations of preimplant site. When patients are not accurately positioned in CBCT scans, CBCT rescan might be necessary for accurate evaluation of preimplant site, and it results in unnecessary radiation exposure to the patient. 

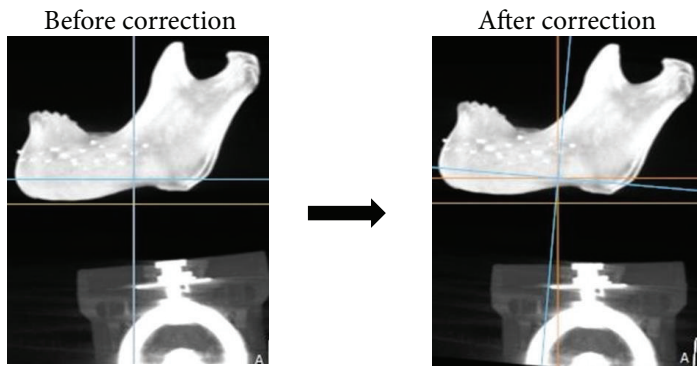

(a)
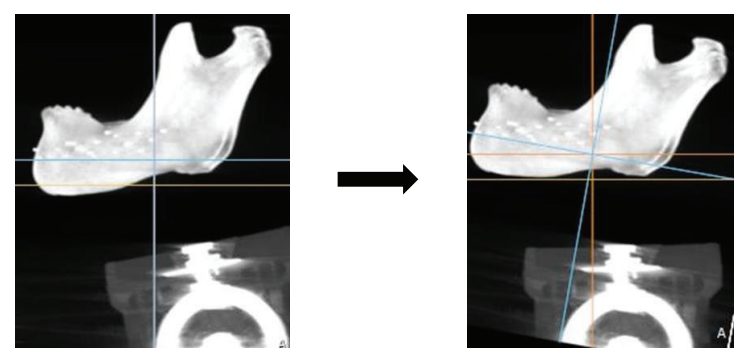

(b)
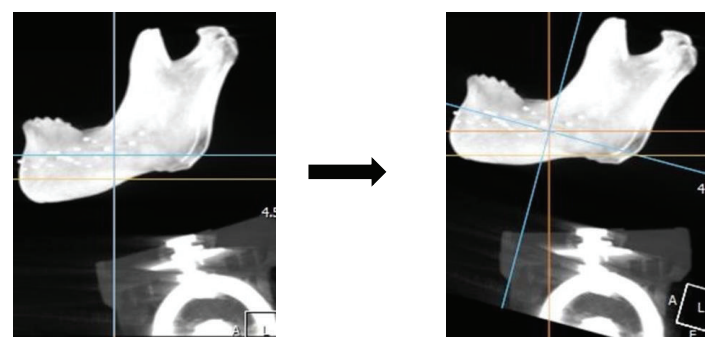

(c)
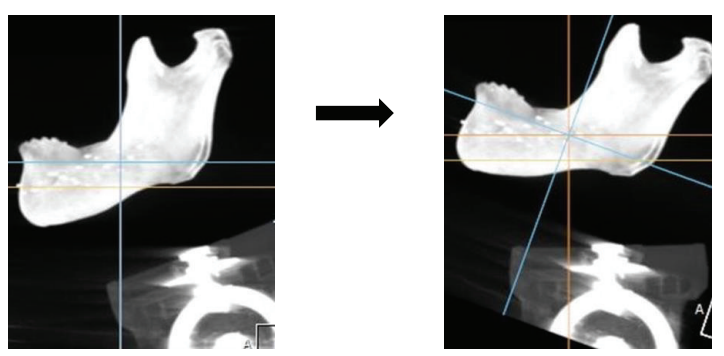

(d)

Figure 3: CBCT images before and after the correction of the mandibular planes. The inferior border of the mandible (mandibular plane) was positioned at 5-, 10-, 15-, and 20-degree angles relative to CBCT scanning table, and the mandibular planes at different angles were corrected to the 0 -degree position using the software. (a) Fivedegree angle location. (b) Ten-degree angle location. (c) Fifteendegree angle location. (d) Twenty-degree angle location.

Today, imaging software has been developed for dental treatment, and the software has the functionality to rotate the axis of CBCT image. We thought that this function should be used for the correction of mandibular plane on all CBCT images that were at angles other than the 0-degree angle. After correction, the vertical measurements at different angles corresponded relatively well with those at the 0 -degree angle. Additionally, a strong correlation was found in the vertical measurements between the 0-degree angle and the other angles in all sites of the mandible. Therefore, the correction of the mandibular plane using software is thought to be a reliable tool for the accurate measurements of the vertical distance in preimplant site of CBCT images.

In conclusion, changes of mandibular position in CBCT scan affected the vertical measurements from the crosssectional images according to the sites of the mandible. However, when CBCT scans are performed at angles other than the 0-degree angle, software-based correction of the mandibular plane can provide satisfactory information about the vertical measurements without requiring an additional CBCT scanning.

\section{Conflict of Interests}

The authors declare that there is no conflict of interests regarding the publication of this paper.

\section{References}

[1] H. H. Zadeh, "Implant site development: clinical realities of today and the prospects of tissue engineering," Journal of the California Dental Association, vol. 32, no. 12, pp. 1011-1020, 2004.

[2] M. G. P. Cavalcanti, J. Yang, A. Ruprecht, and M. W. Vannier, "Accurate linear measurements in the anterior maxilla using orthoradially reformatted spiral computed tomography," Dentomaxillofacial Radiology, vol. 28, no. 3, pp. 137-140, 1999.

[3] M. Halperin-Sternfeld, E. E. Machtei, and J. Horwitz, "Diagnostic accuracy of cone beam computed tomography for dimensional linear measurements in the mandible," The International Journal of Oral \& Maxillofacial Implants, vol. 29, no. 3, pp. 593599, 2014.

[4] J. Gupta and S. P. Ali, "Cone beam computed tomography in oral implants," National Journal of Maxillofacial Surgery, vol. 4, no. 1, pp. 2-6, 2013.

[5] K. B. Waltrick, M. J. Nunes de Abreu Junior, M. Corrêa, M. D. Zastrow, and V. D’Avila Dutra, "Accuracy of linear measurements and visibility of the mandibular canal of cone-beam computed tomography images with different voxel sizes: an in vitro study," Journal of Periodontology, vol. 84, no. 1, pp. 68-77, 2013.

[6] K. Kamburoğlu, C. Kiliç, T. Özen, and S. P. Yüksel, "Measurements of mandibular canal region obtained by cone-beam computed tomography: a cadaveric study," Oral Surgery, Oral Medicine, Oral Pathology, Oral Radiology and Endodontology, vol. 107, no. 2, pp. e34-e42, 2009.

[7] L.-G. Chen, T. Lundgren, H. Hallström, and F. Cherel, "Comparison of different methods of assessing alveolar ridge dimensions prior to dental implant placement," Journal of Periodontology, vol. 79, no. 3, pp. 401-405, 2008.

[8] K. Abramovitch and D. D. Rice, "Basic principles of cone beam computed tomography," Dental Clinics of North America, vol. 58, no. 3, pp. 463-484, 2014.

[9] M. Loubele, N. Van Assche, K. Carpentier et al., "Comparative localized linear accuracy of small-field cone-beam CT and multislice CT for alveolar bone measurements," Oral Surgery, Oral Medicine, Oral Pathology, Oral Radiology and Endodontology, vol. 105, no. 4, pp. 512-518, 2008.

[10] K.-D. Kim, H.-G. Jeong, S.-H. Choi, E.-H. Hwang, and C.-S. Park, "Effect of mandibular positioning on preimplant site measurement of the mandible in reformatted CT," The International 
Journal of Periodontics \& Restorative Dentistry, vol. 23, no. 2, pp. 177-183, 2003.

[11] P. Tarazona-Álvarez, J. Romero-Millán, D. Peñarrocha-Oltra, M.-Á. Fuster-Torres, B. Tarazona, and M. Peñarrocha-Diago, "Comparative study of mandibular linear measurements obtained by cone beam computed tomography and digital calipers," Journal of Clinical and Experimental Dentistry, vol. 6, no. 3, pp. e271-e274, 2014.

[12] R. Jacobs and M. Quirynen, "Dental cone beam computed tomography: justification for use in planning oral implant placement," Periodontology 2000, vol. 66, no. 1, pp. 203-213, 2014.

[13] V. Braut, M. M. Bornstein, U. Kuchler, and D. Buser, "Bone dimensions in the posterior mandible: a retrospective radiographic study using cone beam computed tomography. Part 2-analysis of edentulous sites," The International Journal of Periodontics and Restorative Dentistry, vol. 34, no. 6, pp. 639647, 2014.

[14] L. Vazquez, Y. Nizamaldin, C. Combescure et al., "Accuracy of vertical height measurements on direct digital panoramic radiographs using posterior mandibular implants and metal balls as reference objects," Dentomaxillofacial Radiology, vol. 42, no. 2, Article ID 20110429, 2013. 


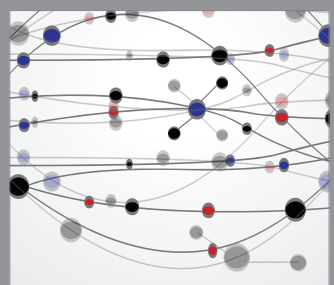

The Scientific World Journal
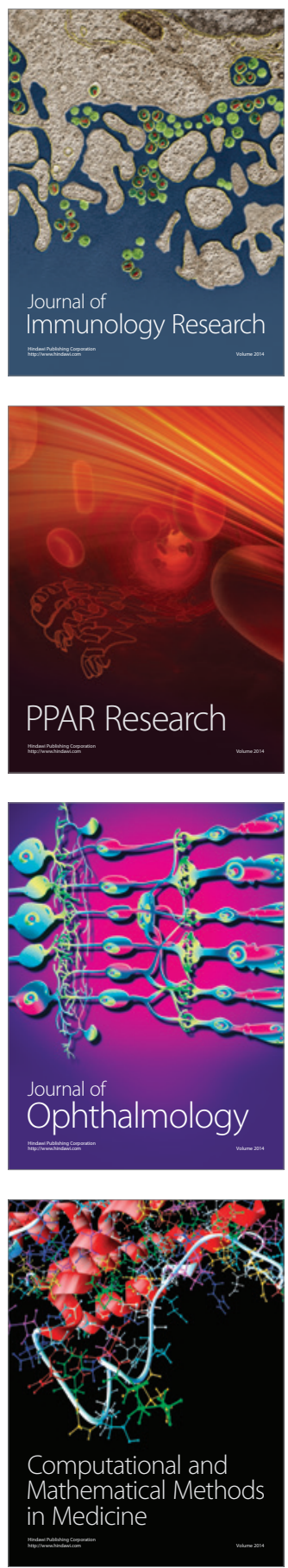

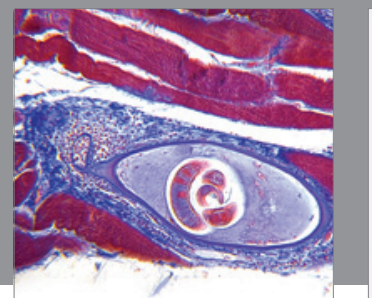

Gastroenterology

Research and Practice
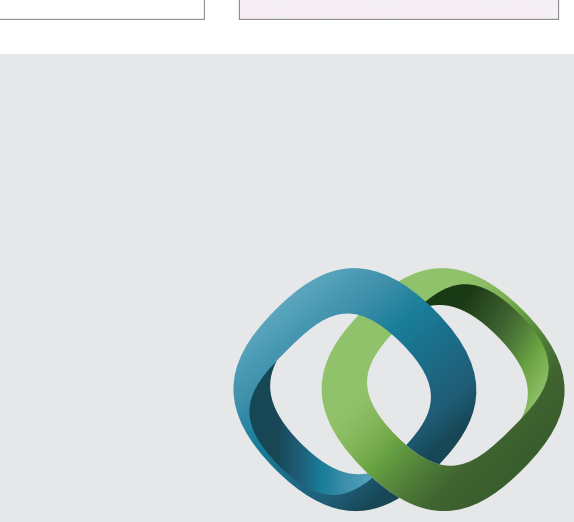

\section{Hindawi}

Submit your manuscripts at

http://www.hindawi.com
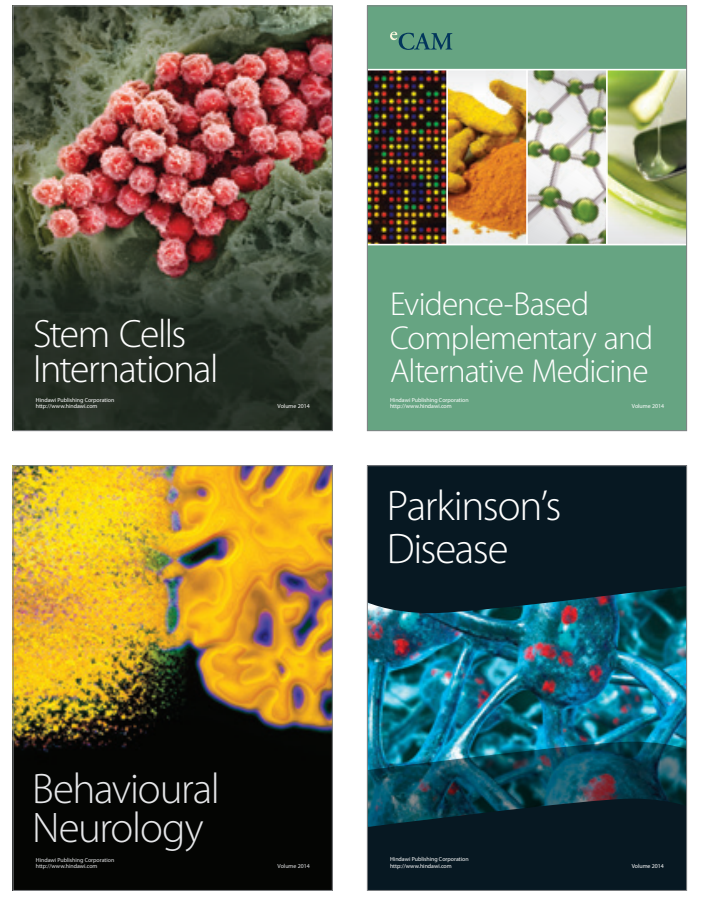
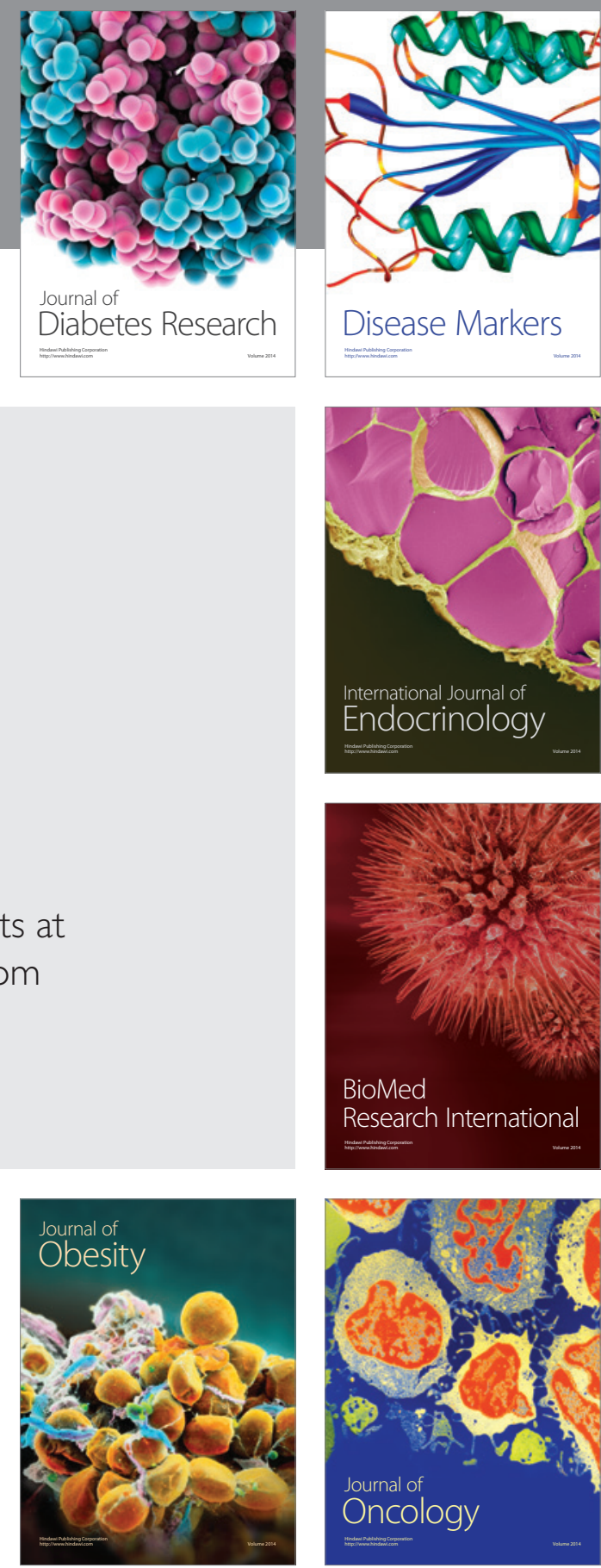

Disease Markers
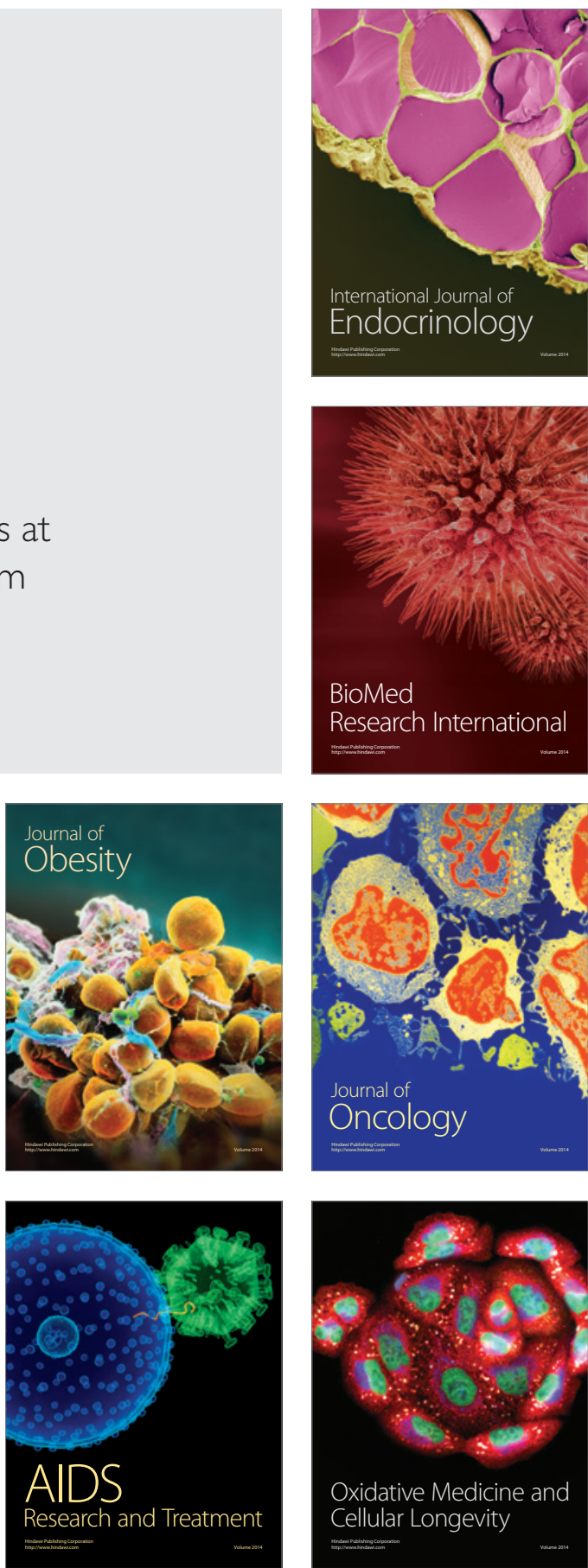